\title{
REAL-TIME MODELING WITH ARCHITECTURAL SPACE
}

\author{
DAVID KURMANN, NATHANEA ELTE AND MAIA ENGELI \\ Architecture and CAAD \\ Swiss Federal Institute of Technology - ETH Zurich, Switzerland \\ [kurmann, elte, engeli]@ arch.ethz.ch
}

Space as an architectural theme has been explored in many ways over many centuries; designing the architectural space is a major issue in both architectural education and in the design process. Based on these observations, it follows that computer tools should be available that help architects manipulate and explore space and spatial configurations directly and interactively.

Therefore, we have created and extended the computer tool Sculptor. This tool enables the architect to design interactively with the computer, directly in real-time and in three dimensions. We developed the concept of 'space as an element' and integrated it into Sculptor. These combinations of solid and void elements - positive and negative volumes - enable the architect to use the computer already in an early design stage for conceptual design and spatial studies. Similar to solids modeling but much simpler, more intuitive and in real-time this allows the creation of complex spatial compositions in 3D space.

Additionally, several concepts, operations and functions are defined inherently. Windows and doors for example are negative volumes that connect other voids inside positive ones. Based on buildings composed with these spaces we developed agents to calculate sound atmosphere and estimate cost, and creatures to test building for fire escape reasons etc.

The paper will look at the way to design with space from both an architect's point of view and a computer scientist's. Techniques, possibilities and consequences of this direct void modeling will be explained. It will elaborate on the principle of humanmachine interaction brought up by our research and used in Sculptor. It will present the possibility to create VRML models directly for the web and show some of the designs done by students using the tool in our CAAD courses. 


\section{Space and Architecture}

\subsection{THE REPRESENTATION OF SPACE IN ARCHITECTURE}

The following section deals with the issue of architectural space representation. A central topic of architectural design is the space, for example in [Tschumi 90]. Every built intervention, even that of a single wall, creates architectural space. The design of a building involves the conceptualisation of complex, interdependent and changing spatial groupings. For a designer, it is important to have a variety of tools available that facilitate modelling, presentation, testing and further development of the spaces and spatial compositions that they conceive.

A few of the traditional methods used for spatial design [Joedicke 85, Van de Ven 87] will be described in order to illustrate the applicability of Sculptor for this aspect of the design task. Sculptor represents a logical extension of these methods and can be used effectively in design.

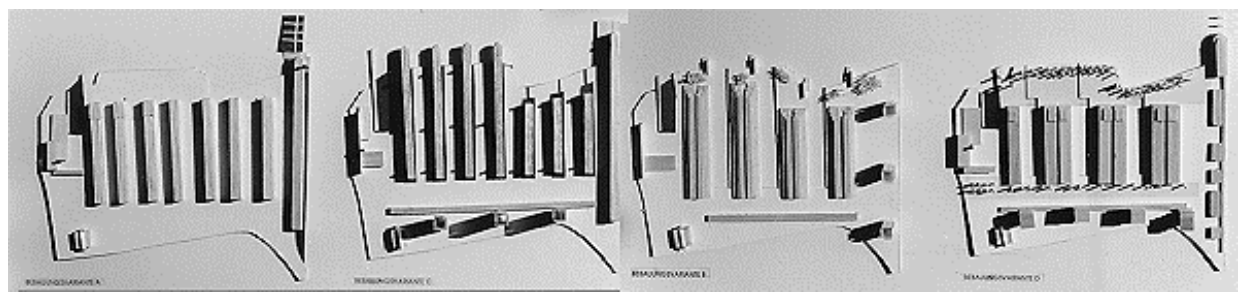

Figure 1. Massing models for volumetric studies

\subsection{VOLUMETRIC REPRESENTATION}

In the first phase of a design project - be it the planning of a new section of a city or the design of single object - massing models are often used to create volumetric studies. Such studies involve the placement of blocks on a site plan or in imagined space. These constellations of blocks represent complex arrangements of spatial elements. When the elements of a massing model are placed during a design process, the exterior space of a project are simultaneously delineated. Exterior spaces can also be considered architectural spaces, as they are defined by the existence of architectural elements. By shifting, exchanging and regrouping the elements of a composition, students can observe the effects of these decisions on their design as a whole. Students can learn to recognise the spatial components of their designs by carefully studying such models, photographs and video tapes; which ideally enables them to design more consciously.

These conceptual studies are used to master a given design problem and prepare for the refinement of the design and the accompanying shift in dimension and detail. To be able to develop a design effectively, students must be able to imagine the spatial consequences that their ideas might have. A physical model is often used to efficiently test and express an idea at this stage. Naturally, perspective drawings can also be used for the same purpose. Although it has been seen that perspectives are more often used for presentation purposes and are rarely an effective part of the design process. It is 
very difficult to work with a drawn perspective. However, the possibility to directly and constantly test the spatial consequences of design decisions remains crucial. This capability can strengthen a persons ability to imagine a proposed space, particularly in the case of students who do not yet have actual building experience. They can learn to judge more effectively whether or not the spaces that they have proposed actually fit the conceptual goals of their design.
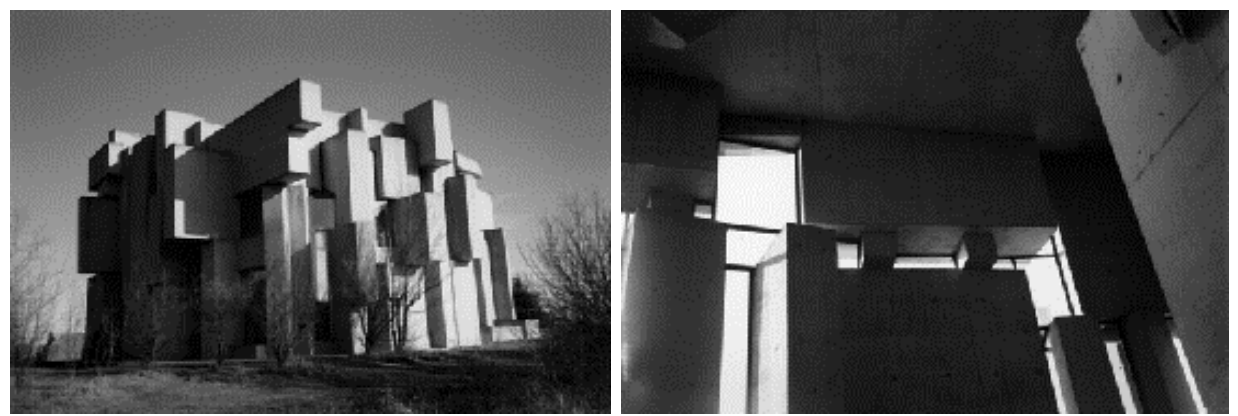

Figure 2. The church 'Zur Heiligsten Dreifaltigkeit' by Fritz Wotruba, complex spaces seen from inside and outside.

\subsection{INTERIOR AND EXTERIOR}

The next step of the refinement process addresses the formal design of individual spaces. A church in Vienna, 'Zur Heiligsten Dreifaltigkeit', by Fritz Wotruba, illustrates how complex spaces can develop and how important the relationships between interior and exterior spaces are. It is difficult to represent the spatial quality of such a church using only plans, sections and a few perspective drawings. Sometimes, students design spatial configurations that are also difficult to visualise, test and present via the more traditional media. However, especially in such cases, this is very important. Additionally, it must be possible to make and visualise changes in a project during the design process. The ability to directly change a design model and to immediately be able to view those changes help the designer develop and judge the design.

There are also spaces that do not involve such expressive massing, but that are nevertheless, very complex. Frank Lloyd Wright's 'Falling Water' is one such example. In it, various spaces and spatial zones - both interior and exterior - blend and overlap throughout the design. Differentiated ceiling design serves to create spatial zoning as well. Similarly, differences in floor levels are also used to express shifts in spatial order. Imagining the spatial aspects of such a project using only the available drawings is not easy. Such spaces can actually only be truly understood when one is able to move about in them; a type of spatial perception through movement. In built projects, one can enhance perception by walking through and around it. For un-built designs, it is valuable to at least be able to simulate such a tour.

A further design concept that has interesting spatial consequences is the development of a facade from the inside out. This approach to facades is based on the idea that a facade not only contribute to and effect the streetscape and spatial aspects of the site and its 
surroundings, but even more strongly the perceptions of the people who use the building. As shown in the example of Wotruba's church, interior and exterior aspects of a design often effect one another very directly. The selected representation method must, if it is truly intended to be used as an instrument of design, clearly represent this direct connection.
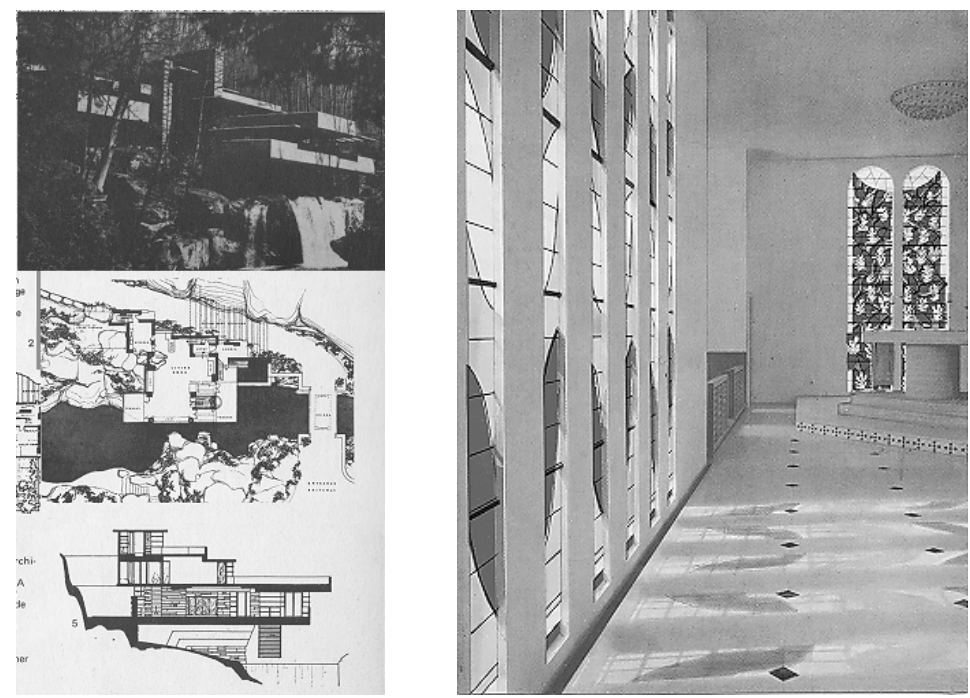

Figure 3. 'Falling Waters' by Frank Lloyd Wright.

Figure 4. 'Chapelle du Rosaire' Henri Matisse

\subsection{SPACE DEFINING ELEMENTS}

The nature of space defining elements, not just the form of a space, effect the perception of a space decisively. One can collect and display material samples, but this, depending on the material, can be quite costly. Being able to simulate the effects of various materials on the perception of a designed space is therefore very desirable and potentially a powerful aid in the design decision making process. The effects and meaning of light in Henri Matisse's 'Chapelle du Rosaire' can, for example, be clearly shown. Being able to simulate such aspects of a design, allows the student to design more exactly and consequently. The implications of light and material on design change constantly during the course of a day. Completely different impressions of a space can occur depending on the time of day. These changes are nearly impossible to convey with drawings, especially when one is attempting to combine it with the total spatial effect of a project. 


\section{Modelling the Space}

\subsection{SPACE AS AN ELEMENT}

This importance of space should be reflected in computer design tool for architecture. Even though this idea is not new - tools that use solid modelling are well known [Mäntylä 88] - we want to propose a slightly different approach. Let's assume that there is a 'space element' ('void element' or 'negative volume'). An element that consists of no material and carves out space when it intersects with a solid element. This would have several interesting and important consequences. The operation with these elements would be different from a solid modeler. Instead of using volumes and boolean operations like subtraction, union, or difference, two types of volumes, positive (solid) and negative (space) volumes are defined. A negative volume always creates a space inside a solid. Where it intersects with a positive volume this becomes visible, but there is no effect where it intersects with another negative volume. This requires also a different data structure and a new description of objects needs to be implemented for CAD. In the following we will show implementation details and results achieved by students using this approach in Sculptor.

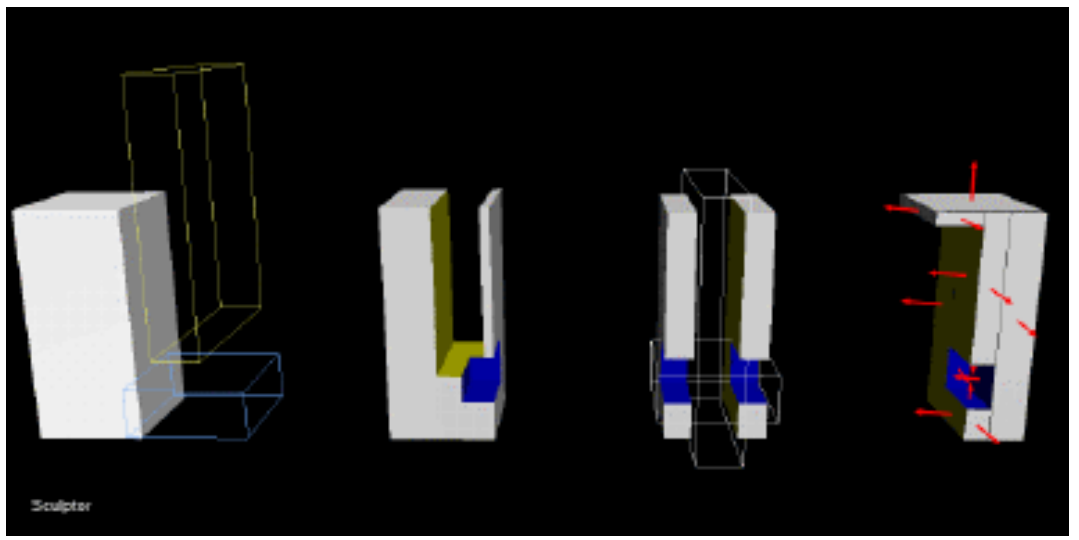

Figure 5. Positive and negative volumes: A sequence of interactively moving two voids (wire frame) into one solid. One special case solved: Entirely separated face of one positive volume. The face normals of the resulting object are indicated on one object.

In our implementation the calculation of objects that are composed of positive and negative volumes is based on four steps: 1) finding out which faces get intersected, 2) cutting each face according to the intersection lines, 3) deciding, whether the trimmed face is shown or not, and 4) finding out, in which direction the face is oriented. Technically, the principle used is a surface modelling technique, used by other authors in a similar way, for example in [Yessios 87].

There are some principles that make this concept easier to realise. A possible face of any composed object will always be a part of an original face of the composing objects. Therefore it does not matter what shape an object has or how it is oriented in space, as long it is described by a closed surface. The intersection of an object with another 
object's face will give the points and lines that define the shape of the resulting face. This trimming process is the most time consuming part of the calculation. The normals of the resulting faces are defined easily: a solid volume will always create faces looking outside, faces looking inside are produced by a void. The fact, that the void defines the corresponding faces makes it possible, that the attribute of a void defines colour and texture of the faces it cuts out.

Two representations, the way an object is composed and the final composition, are stored. This dual representation is needed for two reasons: The final composition is kept because the face calculations should be done as few times as possible. To gain performance, a re-calculation is done only when one of the objects changes. The description of how an object is composed is necessary to do this re-calculation.

This representation of objects is implemented for an orthogonal world with cubes as the only possible voids. The algorithm is generally applicable for every closed object freely oriented in space, but is much faster if it can be reduced to orthogonal cases. The orthogonal world makes interesting and complex objects possible and allows to work with spaces in an intuitive and direct way. With the currently available computing speed, direct manipulation would not be possible in the general case if there are a larger number of volumes in the scene.

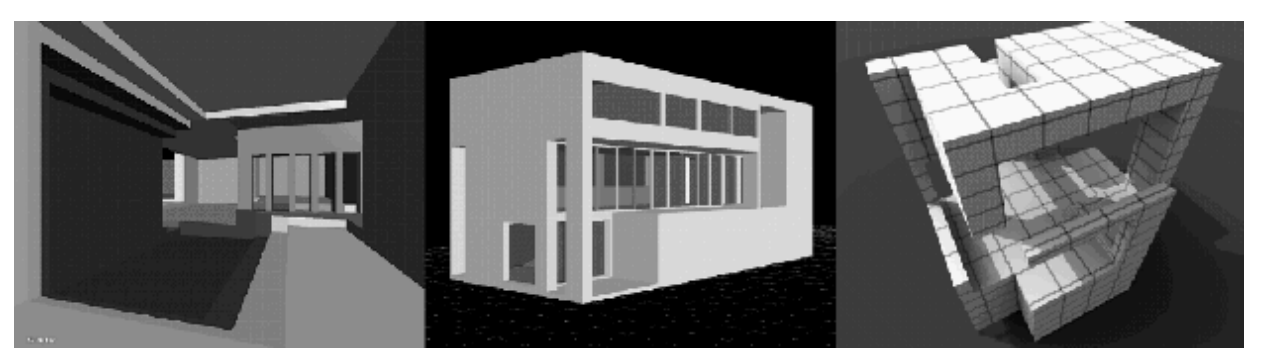

Figure 6. Different architectural models built by using voids and solids in Sculptor: a cave, a rough reconstruction of the 'Casa Cavagli' by Luigi Snozzi and a light simulation rendered with Radiance.

\subsection{ROOMS, WINDOWS, DOORS, AND SECTIONS}

With this concept of negative volumes, the relevant objects and operations for an architect in an abstract phase are defined. Windows and doors are negative volumes that connect other voids inside a positive one. A room can be seen as a composition of a void within a solid. This group then can be intersected with other room groups interactively, a correct composition is always assured. While resizing a room group (a pair of a solid and a void) the thickness of a wall stays constant. Using the negative volume concept, sections through buildings are possible by using a large void and intersecting it with the building. 


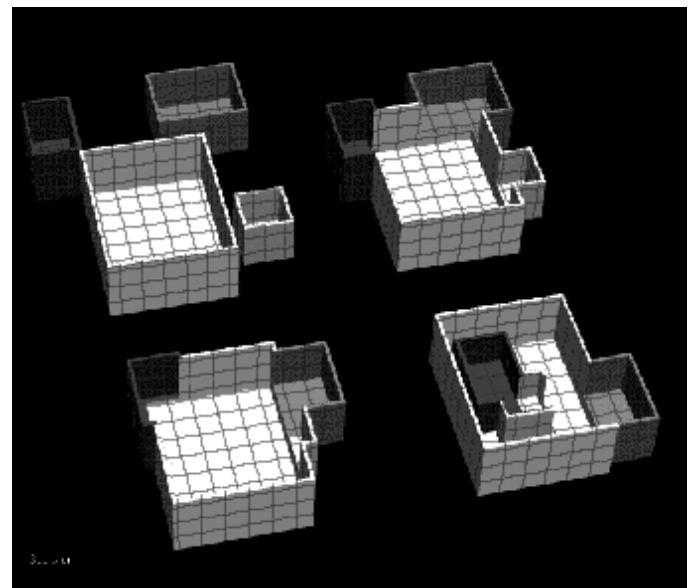

Figure 7. A sequence of how to compose a room (= group of a void and a solid). The rule also allows hierarchical 'room in room' buildings.

Besides the ease of manipulation, the introduction of rooms offers new possibilities in the analysis of buildings. By defining rooms that compose a building (instead of the walls that create the rooms), it is easy to retrieve data for additional support such as: navigation in a building, spatial sound effects, cost estimations, or energy consumption calculations. This will be emphasised later in this article in the description of some intelligent agents.

\section{The Design Tool Sculptor - Space Interactive}

This approach of using 'void element' has been implemented to prove and demonstrate that it works. We wanted to show that interactively creating and manipulating spaces using our approach is more intuitive. Therefore we added this approach to Sculptor, our computer tool for virtual design that has been developed over the last four years [Kurmann 95]. It combines these novel spatial features and functions of the void elements with general concepts of modelling in 3D and focuses on the early, conceptual stage [Van Vries 90] of the architectural design.

A key factor in the program is the human machine interaction. In contrast to many existing CAD tool, Sculptor allows very direct, intuitive and immersive access to three dimensional design models. Through interactive modelling in a virtual space and this introduction of positive and negative volumes, an easy way of generating and manipulating architectural models is made possible. Interactive parameter specification of objects, and models with attributes like form, geometry, colour, or material are supported. Objects can be grouped together hierarchically. Objects, groups and virtual worlds can be changed in real time by scaling, resizing, rotating, reshaping and moving them in space. [Laseau 89]

Different points of view can be chosen as well as functions invoked for walking and flying through $3 \mathrm{D}$ space. All manipulations happen immediately by moving the mouse 
or one of the possible 3D input devices and the scenes are changed and rendered in real time. Multiple windows, complex text input and sliders or buttons are avoided. The interface is almost widget-less.

A direct approach also enables inspiration and creativity when computers are used in design. A direct visual control of what is being designed is critical. It facilitates the development of new ideas, allows a designer to inspect design ideas from any point of view and to discover new solutions to a design problem. It also enables modeling while being inside this architectural virtual space. Sculptor offers the possibility to visualise design, but does not dictate a specific architectural language.
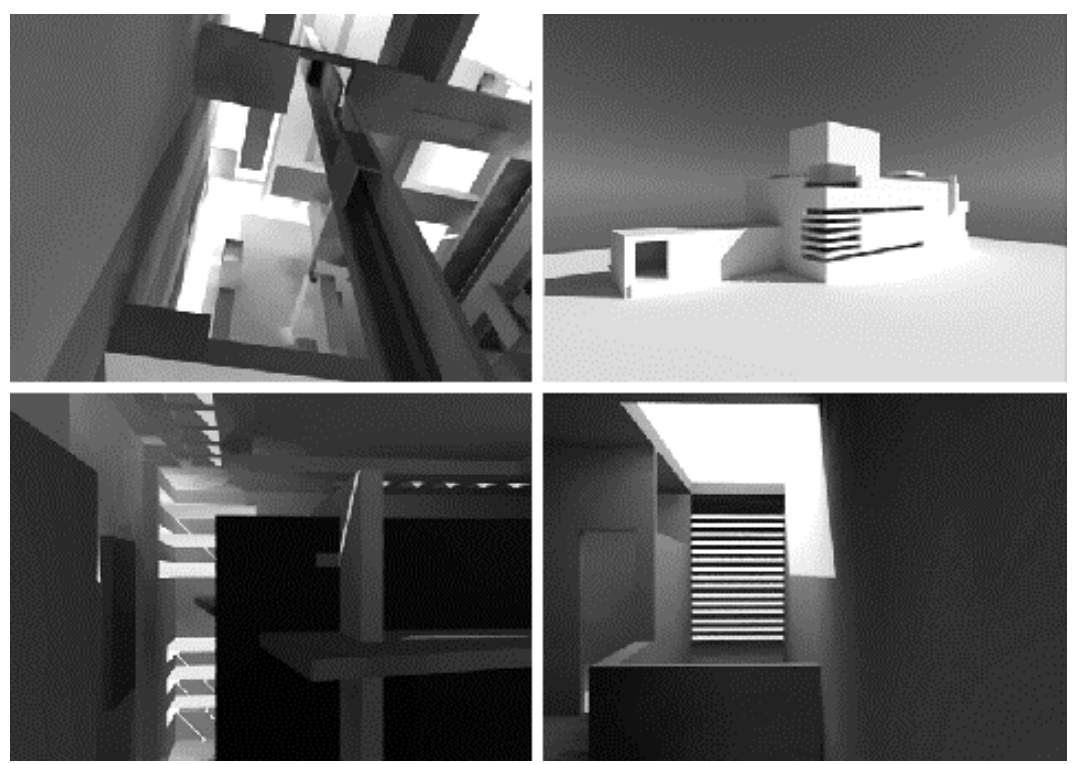

Figure 8. A collection of models that were created by our students in the CAAD courses showing very different conceptions of space and architecture. Images by Habegger, Chladek, Papanikolaou and Nielsen.

Besides assisting the users when creating the three-dimensional geometry of objects, Sculptor also supports models of behaviour based on principles of mechanics and dynamics [Barzel 92]. One is collision detection while objects are changed in size or moved in the scene. Another is the concept of gravity: an object will fall down if it is not supported by another object or standing on the ground. Using these constraints, the interaction with objects in the virtual worlds is enhanced since users experience in a direct way the act of moving objects to valid positions or combining them by following physical principles. Different sorts of feedback are an important feature of virtual reality tools to understand complex scenes in space.

Sculptor designs can be exported to CAD construction tools like AutoCAD for further refinement as well as to VRML files for the World Wide Web. Light simulations with Radiance are possible as shown in figure 8. 


\section{Agents and Space}

We were implementing two basic kinds of agents using these space modeling approach: Design assisting agents, that help the user by providing information and executing background tasks, and design generating agents, that interfere with the design and autonomously come up with new solutions. We started working on design assisting agents first and implemented three prototypical interface agents [Kozierok et al 93]: The Navigator, the Sound Agent and the Cost Agent. They are meant to be personal assistants, trained by each user to adapt to one's individual preferences. All of these agents are based on and take advantage of this data structure which describes the openings and the different kinds of rooms as negative volumes with attributes like room type, size, colour, etc.

The Navigator acts like a guide in the virtual world. It can follow different kinds of instructions like: moving to a specified place, room or building, moving in a specific direction, or composing a tour. He understands commands like: go, show, go to, jump to, that describe the action to be taken, followed by a description what to show (this floor, the building), a place to go to (the kitchen, the entrance, the living room), or the direction to be taken (left, right, up, down, forward, backward). The navigator is built directly into Sculptor. From the information of the spatial composition the Navigator builds a graph. According to the command by the user the Navigator can then search the shortest path in this graph and walks through the building without colliding with walls and furniture.(Fig. 10).
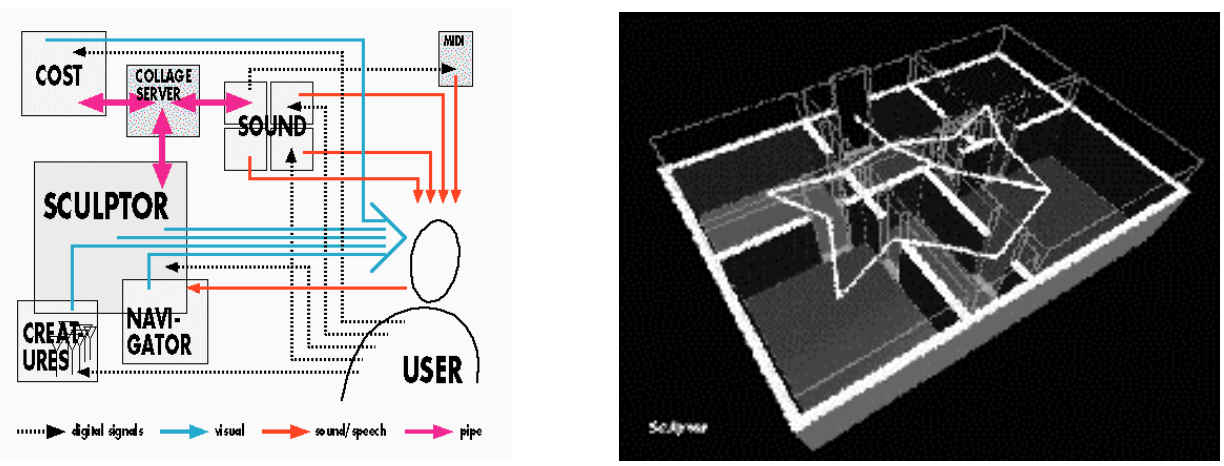

Figure 9. The components and interaction channels of Sculptor \& Agents

Figure 10. The graph built by the Navigator.

The Sound Agent is a companion of the Navigator. It will try to enhance the perception of a space by adding an auditory component to the visual impression. It can play "sound labels", generate the sound of footsteps, change the spatial sound effects, and play a sound track, in accordance to the purpose, shape and colour situation of different rooms. The sound agent is a program that communicates with Sculptor using the NCSA Data Transfer Mechanism (DTM) [NCSA 92]. Sound labels and footsteps can easily be 
generated from the information available from Sculptor. It describes the room the Navigator is in (the purpose, size, and colour situation) and the current position within this room. Which spatial sound effect to use and which sound track to play has to be learned first. [Maes 93]

The Cost Agent estimates the costs of the project and displays the result graphically. The vertical bar represents the costs and turns from green to red when the costs are getting too high. This small interface can be used to define a cost limit, to indicate the desired level of luxury, to access a database of projects and to look at a graph that illustrates the calculation. Like the sound agent the cost agent is a separate program that communicates with Sculptor using the NCSA DTM. Kind and size of each room are considered for the calculations. Using these formulas windows and doors can be treated like rooms. This corresponds to the way the data is represented in Sculptor.

To test the design in different situations, Creatures have been implemented. Creatures are sets of simple agents which can interact with each other and recognise some characteristics of Sculptor models. Their behaviour can be programmed for simulating specific situations, like escaping from a fire, traffic jams or the queue at the cloakroom in a theatre. [Resnik 94]

For the next step we plan to implement agents that connect to information sources that are outside of the actual design studio, like Controlling Agents, Information Agent, HVAC agent or Illumination Agents. Later design agents, that generate and suggest design solutions, will be added. They will be based on a more knowledge based and holistic approach than can be built into Sculptor's intelligent objects.

\section{Conclusion}

Architectural design consists primarily of a complex play between: solids and voids, the development of, movement through, the connections and relationships between, the refinement of space defining elements, light, function, use and construction. For architects and design, it is important to have the most appropriate tool available for every phase of the design process. Particularly, process oriented tools that can be directly used during the designing phases of an architectural project and not just used as a means for presentation. A direct visual control of what is being designed is critical. It facilitates the development of new ideas, allows a designer to inspect design ideas from any point of view and to discover new solutions to a design problem. Sculptor offers the possibility to visualise design, but does not dictate a specific architectural language. We try to enable modelling while being inside this architectural virtual space.

\section{Acknowledgements}

This research is supported by the Swiss National Science Foundation - Special Program Computer Science. 


\section{References}

Barzel, R. (1992) Physical-Based Modelling for Computer Graphics, Academic Press

Joedicke, J. (1985) Space and Form in Architecture - A Circumspect Approach to the Past, Krämer Verlag, Stuttgart

Kurmann, D. (1995) Sculptor - A Tool for Intuitive Architectural Design, in: CAAD Futures '95 - The Global Design Studio, M. Tan and R. Teh (Eds.), University of Singapore, P. 323-330

Kozierok, R., P. Maes (1993) Learning Interface Agents, in Proc. of the 11th National Conference on Artificial Intelligence, AAAI

Maes, P. (1993) Behaviour-Based Artificial Intelligence, in: Proc. of the 2nd Conference on Adaptive Behaviour, MIT Press

Laseau, P., (1989) Graphic Thinking for Architects and Designers, Van Nostrand Reinhold, New York

Mäntylä, M.(1988) An Introduction to Solid Modeling, Principles of Computer Science Principles; 13, Computer Science Press, Maryland

NCSA (1992) Data Transfer Mechanism Programming Manual, ftp://ftp.ncsa.uiuc.edu/DTM/dtm.manual.ps, National Center for Supercomputing Applications

Resnik, M. (1994) Turtles, Termites and Traffic Jams, Complex Adaptation Systems Series, MIT Press, Cambridge

Tschumi, B. (1990) Questions of space - Lectures on Architecture, Architectural Association, London

Van de Ven, C. (1987) Space in Architecture: The Evolution of a New Idea in the Theory and History of the Modern Movements, Van Gorcum, Assen/Maastricht, The Netherlands

Van Vries, M., and H. Wagter, (1990) A CAAD Model for Use in Early Design Phases, The Electronic Design Studio - Architecural Knowledge and Media in the Computer Era. McCullough, Mitchell, Purcell (Eds.), MIT Press, P. 215-228

Yessios, C.I. (1987) The Computability of Void Architectural Modelling, in: Computability of Design Principles of Computer-Aided Design, Y.E. Kalay, ed., Wiley-Interscience, New York, 141-172. 